\title{
ON AN EXTREMAL PROBLEM
}

\author{
PAUl G. NEVAI ${ }^{1}$
}

\begin{abstract}
Let $X=\left(x_{1}, x_{2}, \ldots, x_{N}\right), f: \mathbf{R} \rightarrow \mathbf{C}$ and let $\mathbf{P}_{n}$ be the class of polynomials of degree at most $n$. The generalized Christoffel function $\Lambda_{n}$ corresponding to the measure $d \alpha$ is defined by$$
\Lambda_{n}(X ; f, N, d \alpha)=\min _{\substack{\pi \in \mathbf{P}_{n-1} \\ \pi\left(x_{i}\right)=f\left(x_{i}\right) \\ i=1,2, \ldots, N}} \int_{-\infty}^{\infty}|\pi(t)|^{2} d \alpha(t) .
$$

It is shown that if $\alpha$ satisfies some rather weak conditions then $\lim _{n \rightarrow \infty} n \Lambda_{n}(X ; f, N, d \alpha)$ exists and the limit is also evaluated.
\end{abstract}

The purpose of the present note is to investigate the asymptotic behavior of the functions $\Lambda_{n}: \mathbf{R}^{N} \rightarrow \mathbf{R}$ defined by

$$
\Lambda_{n}(X ; f, N, d \alpha)=\min _{\substack{\pi \in \mathbf{P}_{n-1} \\ \pi\left(x_{i}\right)=f\left(x_{i}\right) \\ i=1,2, \ldots, N}} \int_{-\infty}^{\infty}|\pi(t)|^{2} d \alpha(t) .
$$

Here $X=\left(x_{1}, x_{2}, \ldots, x_{N}\right), f: \mathbf{R} \rightarrow \mathbf{C}$ is a fixed and almost everywhere finite function, $\mathbf{P}_{n}$ is the set of all polynomials $\pi$ of a degree at most $n$ and $\alpha$ is a weight function, that is $\alpha$ is nondecreasing on $\mathbf{R}$, it has infinitely many points of increase and every polynomial $\pi$ belongs to $L_{d \alpha}^{2}$. Therefore $\Lambda_{n}$ is defined and finite for almost every $X \in \mathbf{R}^{N}$.

Estimates for $\Lambda_{n}$ lead to several results in probability theory, statistics and in the theory of orthogonal polynomials. (See [1], [2] and [4].) In fact, it is not hard to explicitly compute $\Lambda_{n}$ [3] but the formula for $\Lambda_{n}$ is so complicated that it cannot be used to estimate $\Lambda_{n}$ when $\alpha$ is not nice. It will be shown that $\lim _{n \rightarrow \infty} n \Lambda_{n}(X ; f, N, d \alpha)$ exists under rather weak assumptions on $\alpha$ and the corresponding limit will also be calculated.

Let $\left\{p_{n}(d \alpha)\right\}_{n=0}^{\infty}$ be the system of polynomials which is orthogonal with respect to $d \alpha$, that is $p_{n}(d \alpha, x)=\gamma_{n} x^{n}+\ldots$ with $\gamma_{n}>0$ and

$$
\int_{-\infty}^{\infty} p_{n}(d \alpha, t) p_{m}(d \alpha, t) d \alpha(t)=\delta_{n m}
$$

Let $M$ denote the class of those weights $\alpha$ for which

$$
\lim _{n \rightarrow \infty} \int_{-\infty}^{\infty} t p_{n}^{2}(d \alpha, t) d \alpha(t)=0
$$

Received by the editors February 14, 1977.

AMS (MOS) subject classifications (1970). Primary 42A52.

Key words and phrases. Orthogonal polynomials, Christoffel functions.

1 Sponsored by the United State Army under Contract \#DAAG29-75-C-0024, and by the National Science Foundation under Grant \#MCS 75-06687 \#3. 
and

$$
\lim _{n \rightarrow \infty} \int_{-\infty}^{\infty} t p_{n-1}(d \alpha, t) p_{n}(d \alpha, t) d \alpha(t)=\frac{1}{2}
$$

Let us remark $M$ contains many weights. If, for instance, $\operatorname{supp}(d \alpha)=[-1,1]$ and $\log \alpha^{\prime}(\cos \theta) \in L^{1}$ then $\alpha \in M$ [5]. Furthermore, if $\alpha$ is absolutely continuous,

$$
\operatorname{supp}(d \alpha)=[-1,1] \text { and } \alpha^{\prime}(t)=\varphi(t) \exp \left\{-\left(1-t^{2}\right)^{-1 / 2}\right\}
$$

where $0<c \leqslant \varphi(t)$ for $-1 \leqslant t \leqslant 1$ and $\varphi$ is Riemann integrable then also $\alpha \in M$ [4]. Another example is the Pollaczek weight [5]. In the following $\Delta$ denotes an interval. It is known that if $\alpha \in M$ then $\Delta \subset \operatorname{supp}(d \alpha)$ iff $\Delta \subset$ $[-1,1][4]$.

TheOREM. Let $\alpha \in M, \Delta \subset \operatorname{supp}(d \alpha), 1 / \alpha^{\prime} \in L^{1}(\Delta)$. Suppose that there exists a sequence $\left\{\varepsilon_{k}\right\}_{k=1}^{\infty}$ with $\varepsilon_{k}>0, \varepsilon_{k} \searrow 0$ such that for every fixed $k$ the function $\Psi_{k}$, which is defined by

$$
\Psi_{k}(t)=\left[\left(1-\varepsilon_{k}\right)^{2}-t^{2}\right]^{-1 / 2} \log \alpha^{\prime}(t),
$$

belongs to $L^{1}\left(-1+\varepsilon_{k}, 1-\varepsilon_{k}\right)$. Then for almost every $X \in \Delta^{N}$

$$
\lim _{n \rightarrow \infty} n \Lambda_{n}(X ; f, N, d \alpha)=\pi \sum_{i=1}^{N}\left|f\left(x_{i}\right)\right|^{2} \alpha^{\prime}\left(x_{i}\right) \sqrt{1-x_{i}^{2}} .
$$

For $N=1$ the Theorem has been proved in [4]. Combining the case $N=1$ with the following Lemma we immediately obtain the Theorem for every $N$.

LEMMA. If $\alpha \in M$ then

$$
\lim _{n \rightarrow \infty} \frac{\Lambda_{n}(X ; f, N, d \alpha)}{\sum_{i=1}^{N} \Lambda_{n}\left(x_{i} ;\left|f\left(x_{i}\right)\right|, 1, d \alpha\right)}=1
$$

for almost every $X \in[\operatorname{supp}(d \alpha)]^{N}$.

Proof. If $\pi \in \mathbf{P}_{n-1}$ then $\pi$ can be expressed as

where

$$
\pi(x)=\int_{-\infty}^{\infty} \pi(t) K_{n}(d \alpha, x, t) d \alpha(t)
$$

$$
K_{n}(d \alpha, x, t)=\sum_{k=0}^{n-1} p_{k}(d \alpha, x) p_{k}(d \alpha, t) .
$$

If $X$ is given and $\Pi_{i<j}\left(x_{i}-x_{j}\right) \neq 0$ then we can write

$$
\begin{aligned}
& \sum_{i=1}^{N} \Lambda_{n}\left(x_{i} ;\left|\pi\left(x_{i}\right)\right|, 1, d \alpha\right) \\
& \quad=\int_{-\infty}^{\infty} \pi(t)\left[\sum_{i=1}^{N} \operatorname{sign}\left\{\pi\left(x_{i}\right)^{2}\right\} \pi\left(x_{i}\right) \Lambda_{n}\left(x_{i} ; 1,1, d \alpha\right) K_{n}\left(d \alpha, x_{i}, t\right)\right] d \alpha(t) .
\end{aligned}
$$


Using Cauchy's inequality, orthogonality relations and the well-known fact that

$$
K_{n}(d \alpha, x, x)=\Lambda_{n}(x ; 1,1, d \alpha)^{-1},
$$

we obtain

$$
\begin{aligned}
& {\left[\sum_{i=1}^{N} \Lambda_{n}\left(x_{i} ;\left|\pi\left(x_{i}\right)\right|, 1, d \alpha\right)\right]^{2} } \\
& \leqslant \int_{-\infty}^{\infty}|\pi(t)|^{2} d \alpha(t) \cdot\left[\sum_{i=1}^{N} \Lambda_{n}\left(x_{i} ;\left|\pi\left(x_{i}\right)\right|, 1, d \alpha\right)+2 \sum_{j<i}\left|\pi\left(x_{i}\right)\right|\left|\pi\left(x_{j}\right)\right|\right. \\
&\left.\cdot \Lambda_{n}\left(x_{i} ; 1,1, d \alpha\right) \Lambda_{n}\left(x_{j} ; 1,1, d \alpha\right)\left|K_{n}\left(d \alpha, x_{i}, x_{j}\right)\right|\right] .
\end{aligned}
$$

If $\alpha \in M$ then clearly $\operatorname{supp}(d \alpha)$ is compact. Therefore by the ChristoffelDarboux formula there exists a number $C$ depending on $X$ and supp $(d \alpha)$ such that

$$
\left|K_{n}\left(d \alpha, x_{i}, x_{j}\right)\right| \leqslant C\left[\left|p_{n}\left(d \alpha, x_{i}\right) p_{n-1}\left(d \alpha, x_{j}\right)\right|+\left|p_{n-1}\left(d \alpha, x_{i}\right) p_{n}\left(d \alpha, x_{j}\right)\right|\right] .
$$

Hence

$$
\begin{aligned}
& {\left[\sum_{i=1}^{N} \Lambda_{n}\left(x_{i} ;\left|\pi\left(x_{i}\right)\right|, 1, d \alpha\right)\right]^{2} } \\
& \leqslant \int_{-\infty}^{\infty}|\pi(t)|^{2} d \alpha(t)\left[\sum_{i=1}^{N} \Lambda_{n}\left(x_{i},\left|\pi\left(x_{i}\right)\right|, 1, d \alpha\right)\right. \\
&+4 C \sum_{i=1}^{N}\left|\pi\left(x_{i}\right)\right|\left|p_{n}\left(d \alpha, x_{i}\right)\right| \Lambda_{n}(x ; 1,1, d \alpha) \\
&\left.\quad \sum_{i=1}^{N}\left|\pi\left(x_{i}\right)\right|\left|p_{n-1}\left(d \alpha, x_{i}\right)\right| \Lambda_{n}\left(x_{i} ; 1,1, d \alpha\right)\right] .
\end{aligned}
$$

Formula (1) implies that $p_{n-1}^{2}\left(d \alpha, x_{i}\right) \Lambda_{n}\left(x_{i} ; 1,1, d \alpha\right)<1$. Consequently by Cauchy's inequality

$$
\begin{aligned}
& {\left[\sum_{i=1}^{N} \Lambda_{n}\left(x_{i} ;\left|\pi\left(x_{i}\right)\right|, 1, d \alpha\right)\right]^{2} } \\
&<\int_{-\infty}^{\infty}|\pi(t)|^{2} d \alpha(t) \sum_{i=1}^{N} \Lambda_{n}\left(x_{i} ;\left|\pi\left(x_{i}\right)\right|, 1, d \alpha\right) \\
& \cdot\left\{1+4 C \sqrt{N}\left[\sum_{i=1}^{N} \Lambda_{n}\left(x_{i} ;\left|p_{n}\left(d \alpha, x_{i}\right)\right|, 1, d \alpha\right)\right]^{1 / 2}\right\},
\end{aligned}
$$


that is

$$
\begin{aligned}
& \sum_{i=1}^{N} \Lambda_{n}\left(x_{i} ;\left|\pi\left(x_{i}\right)\right|, 1, d \alpha\right) \leqslant \int_{-\infty}^{\infty}|\pi(t)|^{2} d \alpha(t) \\
& \cdot\left\{1+4 C \sqrt{N}\left[\sum_{i=1}^{N} \Lambda_{n}\left(x_{i} ;\left|p_{n}\left(d \alpha, x_{i}\right)\right|, 1, d \alpha\right)\right]^{1 / 2}\right\} .
\end{aligned}
$$

This inequality holds for each $X \in \mathbf{R}^{N}$ such that $\Pi_{i<j}\left(x_{i}-x_{j}\right) \neq 0$. Taking the minimum on the right side over every polynomial $\pi \in \mathbf{P}_{n-1}$ such that $\pi\left(x_{i}\right)=f\left(x_{i}\right)(i=1, x, \ldots, N)$ we obtain

$$
\begin{aligned}
\sum_{i=1}^{N} \Lambda_{n}\left(x_{i} ;\left|f\left(x_{i}\right)\right|, 1, d \alpha\right) \leqslant & \Lambda_{n}(X ; f, N, d \alpha) \\
\cdot & \left\{1+4 C \sqrt{N}\left[\sum_{i=1}^{N} \Lambda_{n}\left(x_{i} ;\left|p_{n}\left(d \alpha, x_{i}\right)\right|, 1, d \alpha\right)\right]^{1 / 2}\right\}
\end{aligned}
$$

It has been proved in [4] that if $\alpha \in M$ then for every $x \in \operatorname{supp}(d \alpha)$

$$
\lim _{n \rightarrow \infty} \Lambda_{n}\left(x ;\left|p_{n}(d \alpha, x)\right|, 1, d \alpha\right)=0 .
$$

Consequently, if $\alpha \in M$ then for almost every $X \in[\operatorname{supp}(d \alpha)]^{N}$

$$
\sum_{i=1}^{N} \Lambda_{n}\left(x_{i} ;\left|f\left(x_{i}\right)\right|, 1, d \alpha\right) \leqslant[1+\sigma(1)] \Lambda_{n}(X ; f, N, d \alpha)
$$

where $\lim _{n \rightarrow \infty} \sigma(1)=0$ for almost every $X \in[\operatorname{supp}(d \alpha)]^{N}$. Our next aim is to establish the converse inequality. For a given $X$ let $\pi^{X}$ be defined by

$$
\pi^{X}(t)=\sum_{i=1}^{N} f\left(x_{i}\right) \Lambda_{n-N+1}\left(x_{i} ; 1,1, d \alpha\right) K_{n-N+1}\left(d \alpha, t, x_{i}\right) l_{i}(t)
$$

where $\left\{l_{i}\right\}_{i=1}^{N}$ are the fundamental polynomials of Lagrange interpolation corresponding to $\left\{x_{i}\right\}_{i=1}^{N}$. Clearly $\pi^{X} \in \mathbf{P}_{n-1}$ for almost every $X \in \mathbf{R}^{\boldsymbol{N}}$. Furthermore, if $\pi^{X} \in \mathbf{P}_{n-1}$ then $\pi^{X}\left(x_{i}\right)=f\left(x_{i}\right)$ for $i=1,2, \ldots, N$. Let us compute the $L_{d \alpha}^{2}$ norm of $\pi^{X}$. Introducing the notation

$$
G_{n}(d \alpha, g, x)=\Lambda_{n}(x ; 1,1, d \alpha) \int_{-\infty}^{\infty} g(t) K_{n}^{2}(d \alpha, x, t) d \alpha(t)
$$

we have

$$
\begin{aligned}
\int_{-\infty}^{\infty} & \left|\pi^{X}(t)\right|^{2} d \alpha(t)=\sum_{i=1}^{N}\left|f\left(x_{i}\right)\right|^{2} \Lambda_{n-N+1}\left(x_{i} ; 1,1, d \alpha\right) G_{n-N+1}\left(d \alpha, l_{i}^{2}, x_{i}\right) \\
& +2 \sum_{i<j} \operatorname{Re}\left[f\left(x_{i}\right) \overline{f\left(x_{j}\right)}\right] \Lambda_{n-N+1}\left(x_{i} ; 1,1, d \alpha\right) \Lambda_{n-N+1}\left(x_{j} ; 1,1, d \alpha\right) \\
& \cdot \int_{-\infty}^{\infty} l_{i}(t) l_{j}(t) K_{n-N+1}\left(d \alpha, t, x_{i}\right) K_{n-N+1}\left(d \alpha, t, x_{j}\right) d \alpha(t) .
\end{aligned}
$$


If $\alpha \in M, g$ is continuous on $\operatorname{supp}(d \alpha)$ and $x \in \operatorname{supp}(d \alpha)$ then

$$
\lim _{n \rightarrow \infty} G_{n}(d \alpha, g, x)=g(x)
$$

(see [4]). In particular, if $X$ is such that $\Pi_{i<j}\left(x_{i}-x_{j}\right) \neq 0$ then

$$
\lim _{n \rightarrow \infty} G_{n}\left(d \alpha, l_{i}^{2}, x_{i}\right)=l_{i}^{2}\left(x_{i}\right)=1 \text {. }
$$

Let

$$
I_{i j}=\int_{-\infty}^{\infty} l_{i}(t) l_{j}(t) K_{n-N+1}\left(d \alpha, t, x_{i}\right) K_{n-N+1}\left(d \alpha, t, x_{j}\right) d \alpha(t) .
$$

If $\Pi_{k<l}\left(x_{k}-x_{l}\right) \neq 0$ then $l_{i} l_{j} \in \mathbf{P}_{2 N-2}$. By a direct calculation we obtain that

$$
\begin{aligned}
I_{i j}= & \int_{-\infty}^{\infty} l_{i}(t) l_{j}(t)\left[K_{n-3 N+3}\left(d \alpha, x_{i}, t\right)+\sum_{l=n-3 N+3}^{n-N} p_{k}\left(d \alpha, x_{i}\right) p_{k}(d \alpha, t)\right] \\
& \cdot K_{n-N+1}\left(d \alpha, x_{j}, t\right) d \alpha(t)=l_{i}\left(x_{j}\right) l_{j}\left(x_{j}\right) K_{n-3 N+3}\left(d \alpha, x_{i}, x_{j}\right) \\
& +\sum_{k=n-3 N+3}^{n-N} p_{k}\left(d \alpha, x_{i}\right) \sum_{l=k-2 N+2}^{n-N} p_{l}\left(d \alpha, x_{j}\right) \\
& \cdot \int_{-\infty}^{\infty} l_{i}(t) l_{j}(t) p_{k}(d \alpha, t) p_{l}(d \alpha, t) d \alpha(t) .
\end{aligned}
$$

Since $l_{i}\left(x_{j}\right)=\delta_{i j}$, we get $i<j$

$$
\left|I_{i j}\right| \leqslant C_{1} \sum_{k=n-3 N+3}^{n-N}\left|p_{k}\left(d \alpha, x_{i}\right)\right| \cdot \sum_{l=k-2 N+2}^{n-N}\left|p_{l}\left(d \alpha, x_{j}\right)\right|
$$

where

$$
C_{1}=\max _{\substack{i=1,2, \ldots, N \\ t \in \operatorname{supp}(d \alpha)}}\left|l_{i}(t)\right|^{2} .
$$

Using the recurrence formula which the orthogonal polynomials satisfy it can easily be seen that for $\alpha \in M(5)$ implies

$$
\left|I_{i j}\right| \leqslant C_{2} \sum_{k=n-N-1}^{n-N}\left|p_{k}\left(d \alpha, x_{i}\right)\right| \sum_{l=n-N-1}^{n-N}\left|p_{l}\left(d \alpha, x_{j}\right)\right|
$$

where $C_{2}$ is independent of $n$. Now applying (1) and (2) the second sum on the right-hand side of (4) can easily be estimated. We get

$$
\int_{-\infty}^{\infty}\left|\pi^{X}(t)\right|^{2} d \alpha(t)=[1+\sigma(1)] \sum_{i=1}^{N}\left|f\left(x_{i}\right)\right|^{2} \Lambda_{n-N+1}\left(x_{i} ; 1,1, d \alpha\right)
$$

for almost every $X \in[\operatorname{supp}(d \alpha)]^{N}$. It has been proved in [4] that if $\alpha \in M$ and $x \in \operatorname{supp}(d \alpha)$ then

$$
\lim _{n \rightarrow \infty} \Lambda_{n-N+1}(x ; 1,1, d \alpha) \Lambda_{n}(x ; 1,1, d \alpha)^{-1}=1
$$

for every fixed $N$. Thus

$$
\Lambda_{n}(X ; f, N, d \alpha) \leqslant[1+\sigma(1)] \sum_{i=1}^{N} \Lambda_{n}\left(x_{i} ;\left|f\left(x_{i}\right)\right|, 1, d \alpha\right)
$$


for almost every $X \in[\operatorname{supp}(d \alpha)]^{N}$. The Lemma follows now from estimates (3) and (6).

\section{REFERENCES}

1. G. Freud, Orthogonal polynomials, Pergamon, New York, 1971.

2. U. Grenander and G. Szegō, Toeplitz forms and their applications, Univ. of California Press, Berkeley, 1958.

3. U. Grenander and M. Rosenblatt, An extension of a theorem of G. Szegö and its application to the study of stochastic processes, Trans. Amer. Math. Soc. 76 (1954), 112-126.

4. P. Nevai, Orthogonal polynomials, Mem. Amer. Math. Soc. (to appear).

5. G. Szegö, Orthogonal polynomials, Amer. Math. Soc.. Colloq. Publ., vol. 23, Amer. Math. Soc., Providence, R. I., 1967; 4th ed. 1975.

Department of Mathematics, The Ohio State University, Columbus, Oho 43210

Department of Mathematics and Mathematics Research Center, University of Wisconsin, MADISON, Wisconsin 53706 